\title{
Avaliação da distribuição do antidepressivo fluoxetina para os pacientes da farmácia Municipal de Lavras - MG
}

\author{
Haiane Costa do NASCIMENTO ${ }^{1}$ \\ Daniela Fernanda de FREITAS 2,3 \\ Edimar Agnaldo MOREIRA ${ }^{4}$
}

1. Discente, Centro Universitário de Lavras, Curso de Graduação em Farmácia Generalista, haianecosta@hotmail.com;

2. Docente, Centro Universitário de Lavras, Curso de Graduação em Farmácia Generalista, danielaffreitas@bol.com.br;

3. Docente, Faculdade de Ciências e Tecnologias de Campos gerais, Curso de Graduação em Farmácia Generalista;

4. Discente, Universidade Federal de Alfenas, Pós Graduação em Ecologia e Tecnologia Ambiental, edi.scarab@gmail.com.

Recebido em: 23/05/2013 - Aprovado em: 10/07/2013 - Disponibilizado em: 15/08/2013

RESUMO. Este trabalho avaliou a quantidade do medicamento fluoxetina distribuído para os pacientes da Farmácia Municipal de Lavras, com o objetivo de verificar a quantidade dispensada no período de 12 meses. Foi realizada análises das receitas retidas na Farmácia Municipal de Janeiro de 2009 a Dezembro de 2009. As receitas eram arquivadas dia a dia e o trabalho ficou restrito em separá-las cronologicamente para realizar a análise de dados. Foram prescritos 94251 comprimidos no período de um ano. Foram observadas variações nas quantidades mensais distribuídas do medicamento entre o período de 12 meses. De acordo com os dados coletados, notou-se o aumento do consumo do antidepressivo fluoxetina, devido ao grande número do medicamento distribuído pela Farmácia Municipal de Lavras. Durante o período de coleta de dados, observou-se que a procura pelo medicamento é maior pelo sexo feminino, na faixa etária de 25 a 40 anos, fato este justificado devido à mulher sofrer maior variação no humor persistentemente rebaixado, associado à tristeza, angústia ou a sensação de vazio e redução na capacidade de sentir satisfação ou vivenciar prazer, quando comparado com o homem.

PALAVRAS-CHAVE. : Fluoxetina. Distribuição. Antidepressivo.

ABSTRACT. This study has evaluated the amount of the drug fluoxetine distributed to patients from the Municipal Pharmacy in Lavras, in order to verify the amount prescribed in the period of 12 months. It was performed analyzes of the prescriptions withheld in the Municipal Pharmacy from January 2009 to December 2009. The prescriptions were filed every day and the work was restricted to separate them chronologically to perform data analysis. It was prescribed 94251 tablets in the period of one year. There were great variations in the monthly quantities of the drug distributed between the 12-month period. According to the data collected, it was noted increasing consumption of the antidepressant fluoxetine, due to the large number of drug distributed by the Municipal Pharmacy in Lavras. During the period of data collection, it was observed that the demand for the drug is higher for females, aged 25 to 40 years, and this fact was justified because women suffer more variation in mood persistently downgraded, associated with sadness, distress or the feeling of emptiness and reduced ability to experience pleasure or feel satisfaction when compared with men.

KEYWORDS. Fluoxetine. Distribution. Antidepressant. 


\section{INTRODUÇÃO}

A Fluoxetina é conhecida como pai dos inibidores seletivos da recaptação de serotonina (ISRS). Em 1988 foi introduzida no mercado pela "Eli Lilly" indústria farmacêutica, como Lilly 110140, ou ptrifluoro-fenoxiphenil N-metil-propilamina.

Este fármaco iniciou uma nova era de tratamentos eficazes e seguros para pacientes com depressão (WONG et al., 1995). Desde então, a fluoxetina "a pílula da felicidade na década de 80 " se tornou o fármaco antidepressivo mais prescrito por todo mundo. Com o passar dos anos seu uso foi aprovado para outras indicações, tais como, transtorno obssesivo-compulsivo, bulimia nervosa e outros (ROSSI et al., 2004).

Tal classe de medicamento tem sido avaliada em diversas pesquisas, seja como antidepressivo, como medicamento auxiliar no tratamento da obesidade e recentemente em testes como auxiliar na melhora da memória. De acordo com dado fornecido pela ANVISA, no ano de 2005 a fluoxetina foi o terceiro medicamento controlado mais receitado no Brasil.

A fluoxetina é indicada especialmente para o tratamento da depressão, contudo outros usos foram estabelecidos através da clínica e de estudos clínicos controlados, sendo também indicada para os transtornos obsessivo-compulsivos, bulimia nervosa, transtorno disfórico pré-menstrual; outros usos: transtorno do pânico, transtorno bipolar, síndrome pré-menstrual e neuropatias (GUIA DE MEDICAMENTOS - 2006).

Segundo Laranjeira (2005), o uso racional de medicamentos psicotrópicos ultrapassou a área de especialidade dos psiquiatras e se transformou em um problema de saúde pública. Foram constatadas por inúmeras pesquisas as enormes distorções nas prescrições dos diversos psicotrópicos, feitas pelas mais diferentes especialidades médicas.

A fluoxetina, um antidepressivo de segunda geração, merece destaque em termos de consumo, já que em 1996 foi o fármaco mais vendido nos Estados Unidos, assim como figurou na lista dos dez medicamentos mais vendidos no mundo até o ano de 2000, representado pelo Prozac ${ }^{\circledR}$ (ARGOS, 2005; JENNER, 2005).

Quanto ao emprego terapêutico, a fluoxetina é efetiva no tratamento da depressão, doença compulsivo-obsessiva, bulimia nervosa e doença disfórica prémenstrual. Outro uso potencial da droga inclui a doença do pânico e a distimia (LACY et al., 2004; MICROMEDEX, 2005). No estudo realizado por Panzer (2005) observa-se que os ISRS, de um modo geral, estão bem estabelecidos no tratamento da ansiedade, já que essa condição patológica é considerada uma manifestação frequente da depressão.

Nos últimos anos, segundo Darling (2005), os antidepressivos têm se mostrado como uma das principais classes de medicamentos comercializadas no mercado farmacêutico mundial 
Com base na ampla prescrição do Cloridrato de fluoxetina, esse trabalho tem por objetivo avaliar a quantidade distribuída deste medicamento no período de 12 meses na farmácia municipal de Lavras - MG.

\section{MATERIAL E MÉTODOS}

O presente projeto foi realizado na Farmácia Municipal do município de Lavras, situada a Rua Dona Inácia, 70, Centro. Foram verificados todos os receituários retidos durante o período de janeiro/2009 a dezembro/2009, referentes à distribuição do medicamento Cloridrato de fluoxetina.

A pesquisa foi submetida ao CEP (Comitê de Ética e Pesquisa) e somente após aprovação pelo CEP, a mesma foi executada conforme resolução da lei 196/96 CNS.

Após a aprovação pelo CEP foi apresentado ao farmacêutico responsável da Farmácia Municipal de Lavras o Termo de Consentimento Livre e Esclarecido (Anexo I TCLE) onde o mesmo foi convidado a participar do estudo através de sua autorização (Anexo II - Termo de autorização da Farmácia Municipal de Lavras - MG) para coleta de dados das receitas retidas, ficando ciente do objetivo do estudo.

Foram realizadas visitas semanais a fim de separar as prescrições do medicamento Cloridrato de fluoxetina, durante o período estipulado, com o objetivo de analisar a quantidade diária e mensal distribuída do medicamento.
As receitas encontravam-se em envelopes onde eram guardadas todas as prescrições distribuídas no dia, assim eram separadas as prescrições de fluoxetina para posterior análise.

Os dados obtidos foram codificados e relacionados em uma planilha, onde foram anotadas as distribuições diárias e depois organizadas em gráficos mensais, para análise dos resultados. A variável analisada foi à quantidade distribuída do fármaco fluoxetina no período de janeiro a dezembro de 2009.

\section{RESULTADOS E DISCUSSÃO}

Foram prescritos 94251 comprimidos no período de janeiro a dezembro de 2009 . Como se pôde observar, as quantidades mensais variaram muito a cada mês (Gráfico 01). Essa variação pode ser justificada, em determinado período, pela falta de medicamento na farmácia, visto que as compras eram realizadas por meio de licitações, tendo ocorrido atraso na entrega do medicamento. Nos gráficos 02 a 13, foram apresentadas as distribuições diárias do medicamento fluoxetina em seus respectivos meses.

Gráfico 01- Distribuição anual.

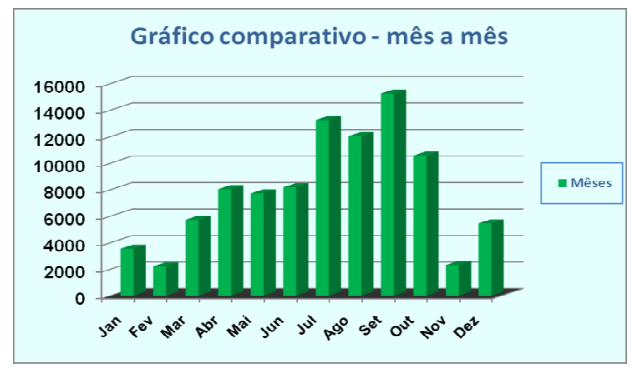

Fonte - Nascimento (2011). 
Durante o ano de 2009, foi verificado um significativo aumento na distribuição de fluoxetina nos meses de julho a outubro, fato que pode ser justificado devido à maior oferta do fármaco fluoxetina neste período.

Analisando os gráficos de janeiro a março percebeu-se um discreto aumento na distribuição do medicamento, mas ainda pouco significativo devido a sua baixa oferta e também em decorrência dos feriados nesta época do ano.

Gráfico 02 - Distribuição diária de medicamento Fluoxetina

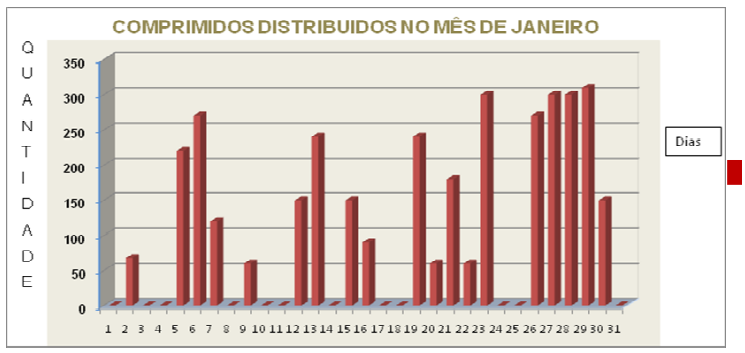

Fonte - Nascimento (2011)

Gráfico 03 - Distribuição diária de medicamento

Fluoxetina.

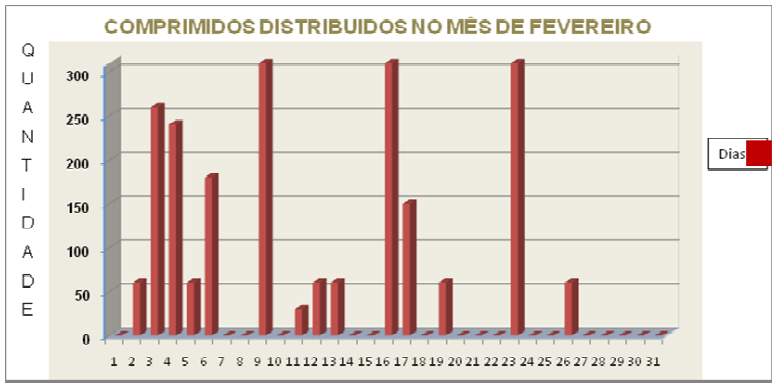

Fonte - Nascimento (2011)
Gráfico 04 - Distribuição diária de medicamento Fluoxetina

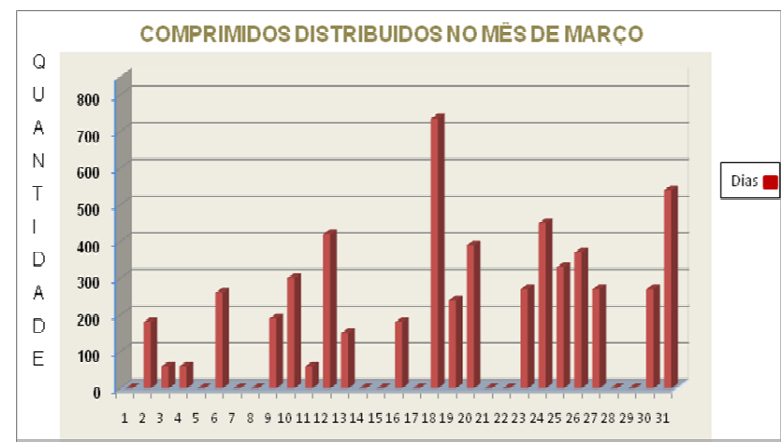

Fonte - Nascimento (2011).

Nos meses de abril a junho houve um aumento significativo na distribuição que se manteve estável durante esse período.

Gráfico 05 - Distribuição diária de medicamento Fluoxetina.

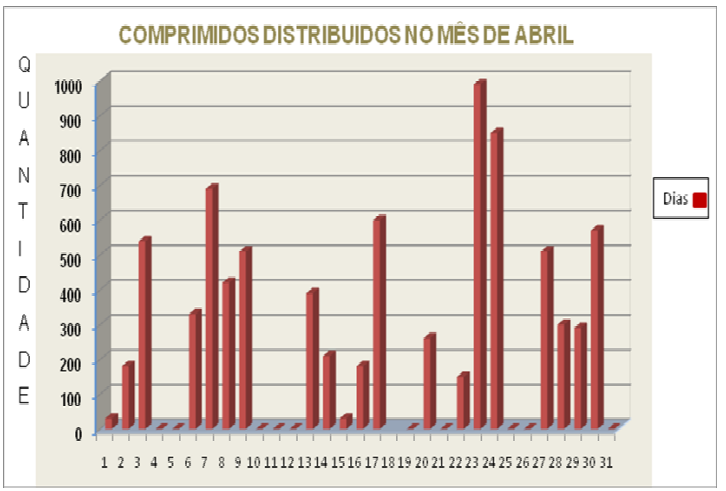

Fonte - Nascimento (2011)

Gráfico 06 - Distribuição diária de medicamento Fluoxetina

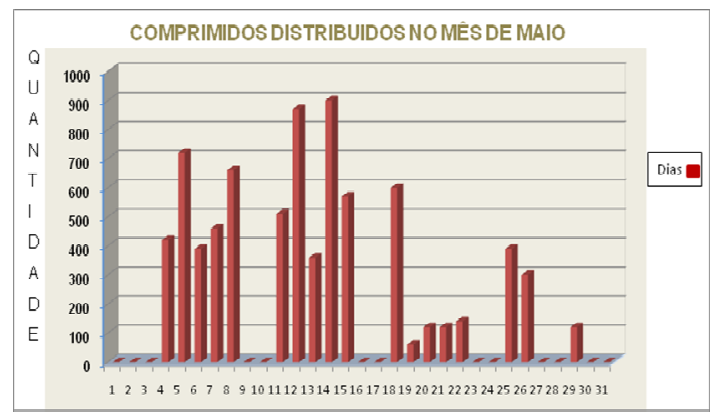

Fonte - Nascimento (2011).

Gráfico 07 - Distribuição diária de medicamento 
Fluoxetina.

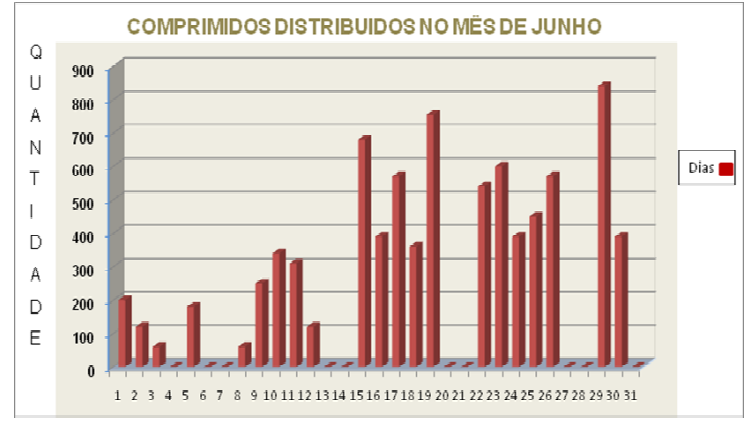

Fonte - Nascimento (2011).

De julho a outubro com a maior oferta do medicamento na farmácia, a distribuição atingiu seu pico máximo com ligeira queda no final do mês de outubro.

Gráfico 08 - Distribuição diária de medicamento Fluoxetina.

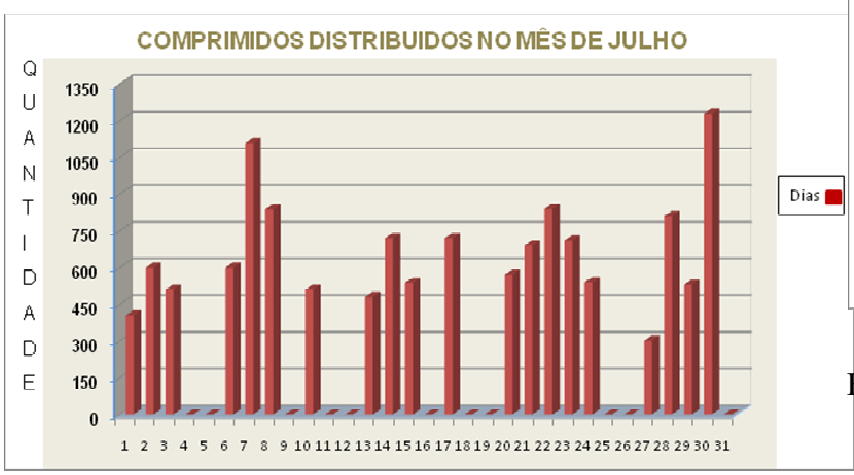

Fonte - Nascimento (2011).

Gráfico 09 - Distribuição diária de medicamento Fluoxetina.

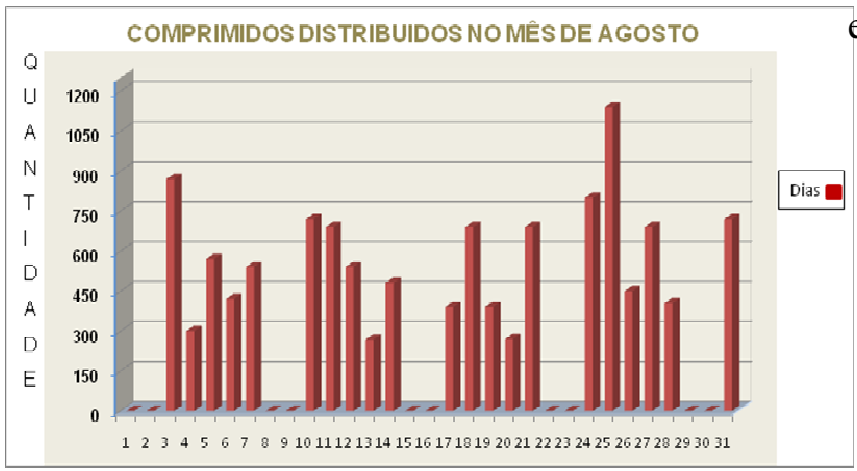

Fonte - Nascimento (2011).

Gráfico 10 - Distribuição diária de medicamento Fluoxetina.

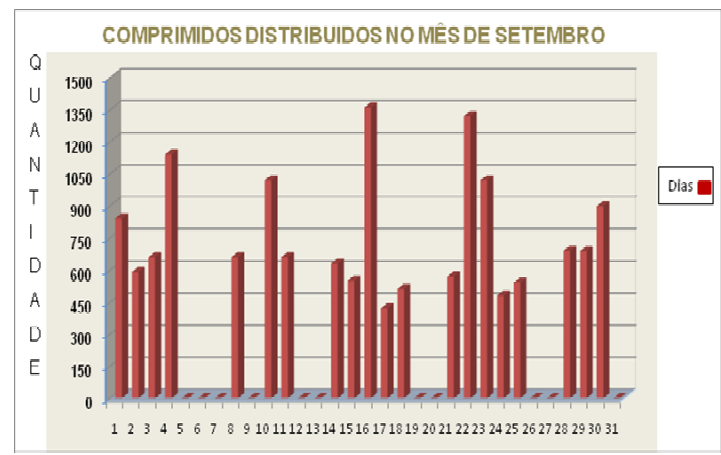

Fonte - Nascimento (2011).

Gráfico 11 - Distribuição diária de medicamento Fluoxetina.

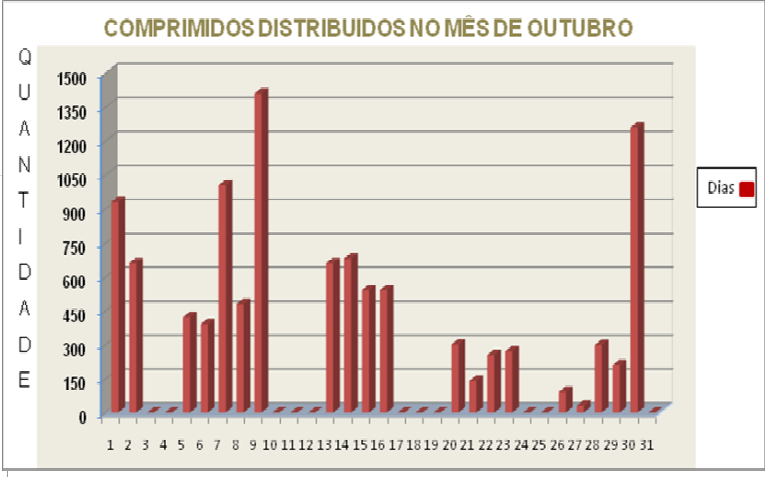

Fonte - Nascimento (2011).

E por fim, pôde-se observar uma queda significativa na distribuição do medicamento. Este fato pode ser justificado por sua pouca oferta nos meses de novembro e dezembro. 
Gráfico 12 - Distribuição diária de medicamento Fluoxetina.

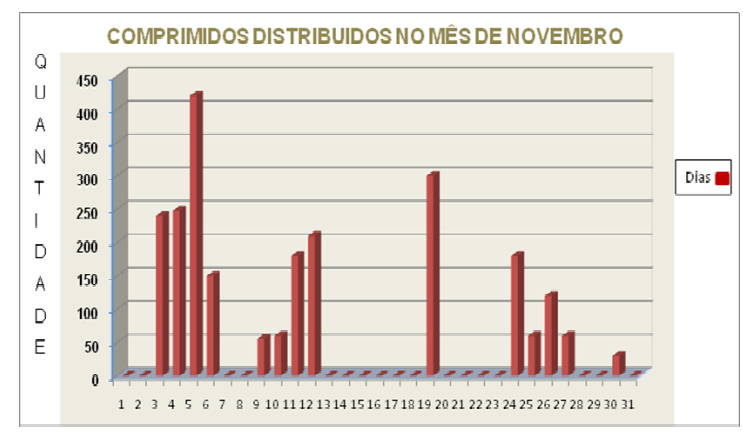

Fonte - Nascimento (2011).

Gráfico 13 - Distribuição diária de medicamento Fluoxetina.

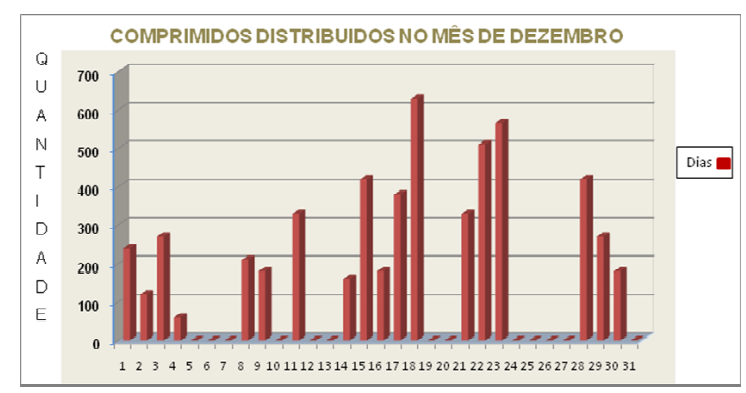

Fonte - Nascimento (2011).

O uso do medicamento fluoxetina foi significativo para o aumento de sua distribuição pela Farmácia Municipal de Lavras. Este aumento só não foi maior, por ser restrita a pacientes em tratamento no CAPS - Centro de Atenção Psicossocial.

Durante o período de coleta de dados, observou-se que a procura pelo medicamento é maior pelo sexo feminino, na faixa etária de 25 a 40 anos. Esta ocorrência maior em mulheres deve-se ao fato de que nelas ocorrem alterações hormonais, como na puberdade, menopausa, período prémenstrual, pós-parto e questões afetivas. O início é precoce em torno dos 20 anos, mas pode ocorrer inclusive em crianças. Idosos são mais vulneráveis pelas limitações biológicas e psicossociais próprias dessa faixa etária (MESQUITA, 2005).

A Fluoxetina mesmo classificada terapeuticamente como antidepressiva possui características anorexígenas e vem sendo prescrita por muitos médicos como mais um subsídio para proporcionar a redução de peso. (CAVALCANTI, 2010/2011).

De acordo com Carlini (2009) a fluoxetina é um ISRS de grande utilidade para o tratamento das depressões, mas pode produzir reações adversas graves, inclusive ideação suicida. De fato, o sistema de psicofarmacovigilância (Psifavi) do Cebrid (Centro Brasileiro de Informações sobre Drogas Psicotrópicas do Departamento de Psicobiologia da Unifesp) analisou, ao longo de 3 anos, 15 notificações de reações adversas produzidas por fluoxetina, descritas por médicos psiquiatras, tais como ideação suicida, bruxismo, elevação das enzimas hepáticas, hiperglicemia, alucinação, metrorragia, sintomas extrapiramidais, amenorréia, tremores etc.

Observou-se ainda neste estudo que, na imensa maioria das prescrições, a fluoxetina foi indicada para mulheres. Esse achado não encontra justificativa médica ao se verificar que na população brasileira a prevalência de depressão, principal indicação da fluoxetina, atinge a proporção de apenas duas mulheres para cada homem. Os dados do estudo realizado por Carlini (2009) mostraram 
uma relação muito maior, de 6,8 mulheres para cada homem que recebeu prescrição de fluoxetina.

Merece ainda destaque o fato de a fluoxetina dispensada nas farmácias ter sido prescrita em associações com outras substâncias em praticamente $100 \%$ das prescrições. Nessas prescrições aparecem substâncias que não são comumente indicadas para as síndromes depressivas. Ao contrário, essas associações estão quase sempre presentes em fórmulas magistrais para indução de anorexia, visando à perda de peso.

$O$ uso da fluoxetina em fórmulas para emagrecer foi até mesmo condenado pela FDA (Food and Drug Administration) dos Estados Unidos. Diz aquele órgão público americano: "Duas pílulas brasileiras para dieta, a 'Emagrece Sim Dietary Supplement' (também conhecida como 'Brazilian Diet Pill') e a 'Herbathin Dietary' Supplement', fabricadas pelos Laboratórios Fitoterápicos (também escrito Fytoterapicos) e Phytotherm Sim, contêm drogas ativas, inclusive substâncias controladas, que podem causar reações adversas sérias ou injúria ao paciente, além do mais, ambos os produtos não foram aprovados pelo FDA". A FDA afirma ainda que ambos os produtos contenham várias substâncias, entre elas, 'a 'fluoxetina', que está ligada a casos de sérias interações com outros medicamentos e a alguns eventos adversos sérios, incluindo ideação e comportamento suicida em crianças, ansiedade, insônia e hemorragia abdominal”.
Acresce-se ainda que a associação da fluoxetina com várias outras substâncias aumentaria a possibilidade de interações farmacológicas desfavoráveis, em virtude da interação com as enzimas do sistema P450. Pode-se, portanto, supor que a redução de peso foi um dos motivos das prescrições da fluoxetina, uma finalidade inadequada, sem uma relação risco - beneficio que as justificassem.

\section{CONCLUSÃO}

\begin{abstract}
De acordo com os dados deste trabalho foi observado uma ampla distribuição de fluoxetina, fato que pode ser associado ao aumento de pessoas que sofrem com a depressão, uma patologia que acomete todas as faixas etárias.
\end{abstract}

Desta forma, concluiu-se que a fluoxetina é muito utilizada em prescrições médicas como antidepressivo e como auxiliar na perda de peso.

A variação observada durante o ano em estudo pode ser justificada pelo fato de sua distribuição ser restrita a pacientes do CAPS - Centro de Atenção Psicossocial.

\section{REFERÊNCIAS BIBLIOGRÁFICAS}

Argos L. En España el Prozac arrasa pese a que existen siete genéricos. Fármacos v. 3, n. 1, p. 11-12, 2005. 
CARLINI, E. A. et al. Fluoxetina: indícios de uso inadequado. Jornal Brasileiro de

Psiquiatria. v. 58, n. 2, p. 97-100, 2009.

CAVALCANTI, N.C.T. et al. Avaliação de prescrições médicas contendo cloridrato de fluoxetina, como agente antiobesidade, em farmácias magistrais. Pharmacia Brasileira. Ano XII, n.79, p.60-64. 2010/2011.

DARLING, L. IMS World Review.2005.

Disponível em: <www.imshealth.com>. Acesso em 20 de março de 2010.

JENNER, P. IMS World Review, 2005 Disponível em: <www.imshealth.com>. Acesso em 10 março de 2010.

LACY C F et al. Drug information handbook. 12. ed. Hudson: Lexi-Comp., 2004, p. 623-625.

LARANJEIRA, R. O uso racional de medicamentos psicotrópicos no Brasil. 2005. Disponível em: <www.uniad.org.br>. Acesso em: 25 de março de 2010.

MESQUITA, M.E. Transtornos depressivos. Rev. SNC em foco, São Paulo, v.1, n.1, p.2027, 2005.

MICROMEDEX . Disponível em:

http://www.dotlib.com.br/micromedex. 2005. Acesso em 10 de março de 2010.

Panzer M. I. Are SSRIs really more effective for anxious depression?. Ann. Clin.

Psychiatry, v. 17, n. 1, p. 23-29, 2005.

ROSSI, A ; BARRACO, A; DONDA, P. Fluoxetine: a review on evidence based medicine. BioMed Central. 2004, p. 1-8. 